\title{
Fiber and Integrated Waveguide-Based Optical Sensors
}

\author{
Valerio Pruneri, ${ }^{1}$ Christos Riziotis, ${ }^{2}$ Peter G. R. Smith, ${ }^{3}$ and Athanasios Vasilakos ${ }^{4}$ \\ ${ }^{1}$ The Institute of Photonic Sciences (ICFO) and ICREA, Mediterranean Technology Park, Avenue del Canal Olimpic s/n, \\ 08860 Castelldefels (Barcelona), Spain \\ ${ }^{2}$ Photonics for Nanoapplications Laboratory, Theoretical and Physical Chemistry Institute, National Hellenic Research Foundation \\ (NHRF), 48 Vassileos Constantinou Avenue, 11635 Athens, Greece \\ ${ }^{3}$ Optoelectronics Research Centre, University of Southampton, Southampton SO17 1BJ, UK \\ ${ }^{4}$ Department of Electrical and Computer Engineering, National Technical University of Athens, 15780 Athens, Greece
}

Correspondence should be addressed to Christos Riziotis, riziotis@eie.gr

Received 15 December 2009; Accepted 15 December 2009

Copyright (c) 2009 Valerio Pruneri et al. This is an open access article distributed under the Creative Commons Attribution License, which permits unrestricted use, distribution, and reproduction in any medium, provided the original work is properly cited.

Over the last years, a large part of the activity in applied photonics and especially in fiber or integrated waveguidebased devices has been transferred partially from the photonics telecommunications industry towards the optical sensors research. Further to the necessity due to telecommunications sector turn down, it has been proven that this shift has been welcomed by the development in relevant industrial sectors (pharmaceutical, medical) where new requirements for very accurate control of the manufacturing process are required. This increasing research effort on all-optical sensors' technology, combined with emerging and demanding applications, has demonstrated a promising technological platform characterized by unique sensitivity, compactness, reliability, electromagnetic immunity, and low cost, promoting them to a preferable solution for real-world applications, from mechanical sensing to chemical/biochemical and pharmaceutical industry. The inherent also capability of photonics technology for the efficient sensing-signal transmission through optical fibers suggests an enhanced functionality from a system's perspective, by enabling the high-speed interconnection of multiple remote sensing points, either through a single readout and administration unit, or through a distributed network. Furthermore the need for development of large-scale ad hoc sensor networks requires reliable autonomous and controllable sensing nodes and optical sensors exhibit very attractive and unique characteristics to play key role in this area. Emerging technologies combining new design concepts and operational approaches such as microstructured fibers (PCFs), tapered nanofibers, Bragg gratings, and long-period gratings, interferometric devices, as well as Surface Plasmon Resonance (SPR) devices have shown a strong impetus for novel applications. A critical issue which could dramatically enhance the performance of such functional devices is the use of novel polymers and nanostructured materials able to improve the sensitivity and expand also sensors selectivity range.

This special issue is completely devoted in this dynamic area of optical sensors, aiming to broadly cover aspects such as material properties, fabrication techniques, modeling, optimization, and novel applications. Hosting 17 representative papers demonstrates successful engineering of novel fiber and planar optical sensors for a variety of applications such as physical, chemical, and biosensing. Selected papers have been invited in order to provide the state of the art and current trends in distinct hot areas.

The issue begins with a group of review papers on key fiber-based sensor categories, with the first invited paper from Gupta and Kumar which gives an up-to-date review on fiber optic-based surface plasmon resonance sensors demonstrating applications in measuring various physical, chemical, and biochemical parameters. Various designs of the fiber optic SPR probe were reported there for the enhancement of sensors' sensitivity. The second invited paper by Skorobogatiy presents a comprehensive review on microstructured optical fiber and photonic bandgap (PBG) fiber-based resonant optical sensors. Two sensor architectures are discussed where in the first one were employed hollow core photonic bandgap fibers where coreguided mode is strongly confined in the analyte-filled core. The second sensor case employed metalized photonic 
bandgap waveguides and fibers, where core-guided mode was phase matched with a plasmon propagating at the metalized fiber/analyte interface. Operational regions of the resonant sensors are reviewed covering a wide range of wavelengths from the visible to terahertz. Canning presents in his invited paper the state-of-the-art work on sensors based on Bragg gratings and microstructured fibers. The paper presents the current situation and the measurements challenges with temperature and strain and discusses as well novel engineering concepts such as an integrated lab in a fiber. Another architecture based on modal interferometers on PCFs is reviewed by Villatoro et al. as a solution for highly sensitive sensors of low thermal sensitivity and applications ranging from strain, temperature, to refractive index and volatile organics. Sagnac, Mach-Zehnder, or Michelson-like interferometers can be fabricated by different postprocessing techniques such as grating inscription, tapering or cleaving, and splicing. Margulis et al. propose in their invited research paper a novel and alternative interferometric architecture based on the plurality of individual fiber preforms drawn together but nearly maintaining their original shape. The potential temperature independence and the ease of splicing of Gemini fibers make them an attractive solution for sensors development in a monolithic-robust multicore fiber design.

The following three papers present architectures and applications of PCFs for physical parameters sensing. The first paper by Ju and Jin is an invited review on photonic crystal fibers utilizing either long-period gratings or in-fiber modal interferometers for strain and temperature sensing. The paper suggests that air-silica PCF sensors are comparable or better than those implemented in conventional singlemode fibers but the temperature sensitivities of the PCF sensors are much lower. Next paper by Larrion et al. presents a novel configuration of a temperature sensor based on a PCF with quantum dot nanocoatings in its inner holes, deposited by means of the Layer-by-Layer technique. The paper studies for a temperature range from -40 to $70^{\circ} \mathrm{C}$ the optical properties of these sensors and introduces also the consideration of the full width at half maximum (FWHM) as a new insightful characterization parameter. Another configuration of a temperature sensor with a lowcost amplitude interrogation technique is presented in the paper by Torres-Peiro et al. based on the cutoff properties of the fundamental mode in a liquid filled Y-shaped Ge-doped microstructured fiber. The sensitivity is mainly determined by the thermo-optic coefficient of the filling liquids and values of $25 \mathrm{~nm} /{ }^{\circ} \mathrm{C}$ with detection limit of about $0.001{ }^{\circ} \mathrm{C}$ are reported.

The next three papers present different approaches for measuring also physical parameters but using conventional fibers. The invited review by Yamashita presents a novel wide and fast wavelength-swept fiber laser architecture for dynamic and accurate fiber sensing, based on the dispersion tuning technique, by modulating the loss/gain in the dispersive laser cavity. Kuang et al. present in their invited review a low-cost platform based on plastic optical fibers-POF for the quite important area of Structural Health Monitoring (SHM). Between different possible schemes, the intensitybased interrogation capability of POF sensors provides additional a very favorable solution of low-cost SHM systems implementation. The next paper by Pinet gives from an industrial and applications perspective the state-of-the-art situation on miniaturized sensors based on Fabry-Perot fiber optic implementation. This alternative architecture provides a mature and reliable technology for strain, temperature, pressure, displacement, or refractive index measurements, and with a lot of applications ranging from industrial to medical.

The following three papers are devoted to fiber optic based chemical and biosensing, where the first invited paper by Dagenais presents a promising sensor based on an etched Bragg grating functionalized with glucopyranosyl-siloxane conjugate in order to interact selectively with glucose binding proteins, altering thus the surface's refractive index. Next paper by Carvalho et al. presents a hollow-core PCF for detecting low levels of methane. A prototype is demonstrated in a portable system incorporating an interrogation scheme based on the Wavelength Modulation Spectroscopy technique. In the next paper by Andres et al., another simple technique based on single mode fiber at $850 \mathrm{~nm}$ is presented, where a long-period grating as an equivalent $3 \mathrm{~dB}$ beam is used to form a coaxial-Michelson modal interferometer. Direct dipping of the sensor head in water solutions permits the measure of small refractive index changes.

The final group of papers is devoted to the integrated optics approach which offers a promising technological platform towards robust and multifunctional sensors physically compatible with microfluidics. The invited paper by Kashyap reviews the state of the art on Surface Plasmon Resonance-based devices focusing especially on their integrated platform approach as a route for low cost, mass production, disposable sensors. The next paper by Sparrow et al. reviews the emerging class of integrated Bragg grating-based sensors, and examines in detail the architecture, fabrication, and applications of special sensors' chips fabricated by the flexible direct UV writing technique on silica-on-silicon wafers. A variety of applications ranging from temperature, refractive index, chemical, and biosensing are described and also indicative specific problems in food industry for monitoring long-term industrial process such as fermentation are demonstrated. The final invited paper is by Mayeh et al. describing another class of integrated devices, based on the operational principle of slotted multimode interference (MMI). The paper presents design and modeling results towards the tuning of the device in order to detect protein-based molecules or water-soluble chemical or biological materials. Fabrication of the device has been demonstrated in silicon oxynitride ( $\mathrm{SiON}$ ) as highly stable to the reactivity with biological agents and processing chemicals.

Introducing this special issue to the Journal of Sensors, we would like to thank all the authors for their prompt and valuable contributions and also the reviewers for their critical help, necessary to achieve a high level of papers' quality and make thus possible the completion of this special issue. Also we would like to thank the Editor in Chief Professor Francisco J. Arregui and the Editorial Board for approving this special issue as well as the Journal's staff for their 
professionalism and effective consideration of all the details during the preparation of the issue.

Valerio Pruneri

Christos Riziotis

Peter G.R. Smith

Athanasios Vasilakos 

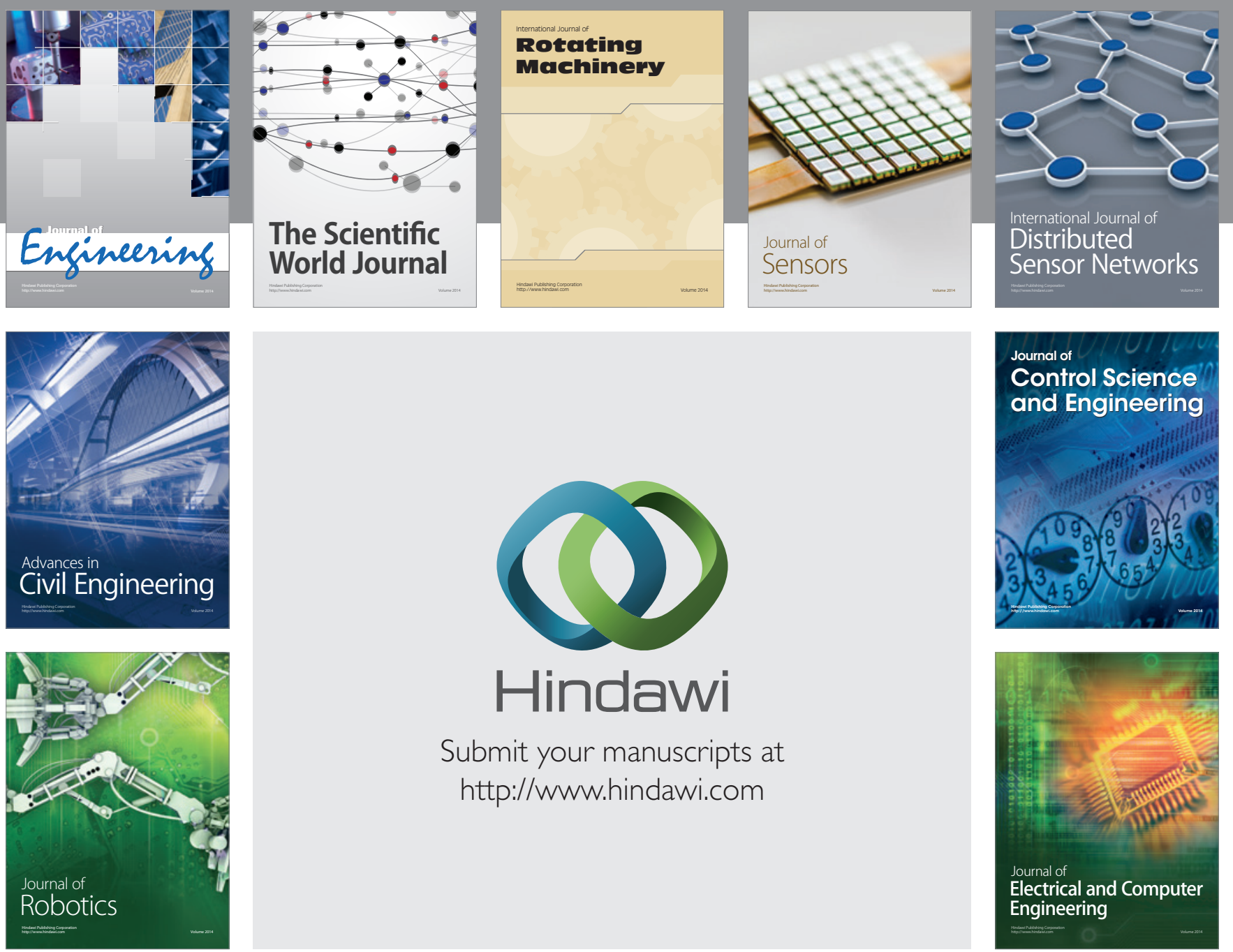

Submit your manuscripts at

http://www.hindawi.com
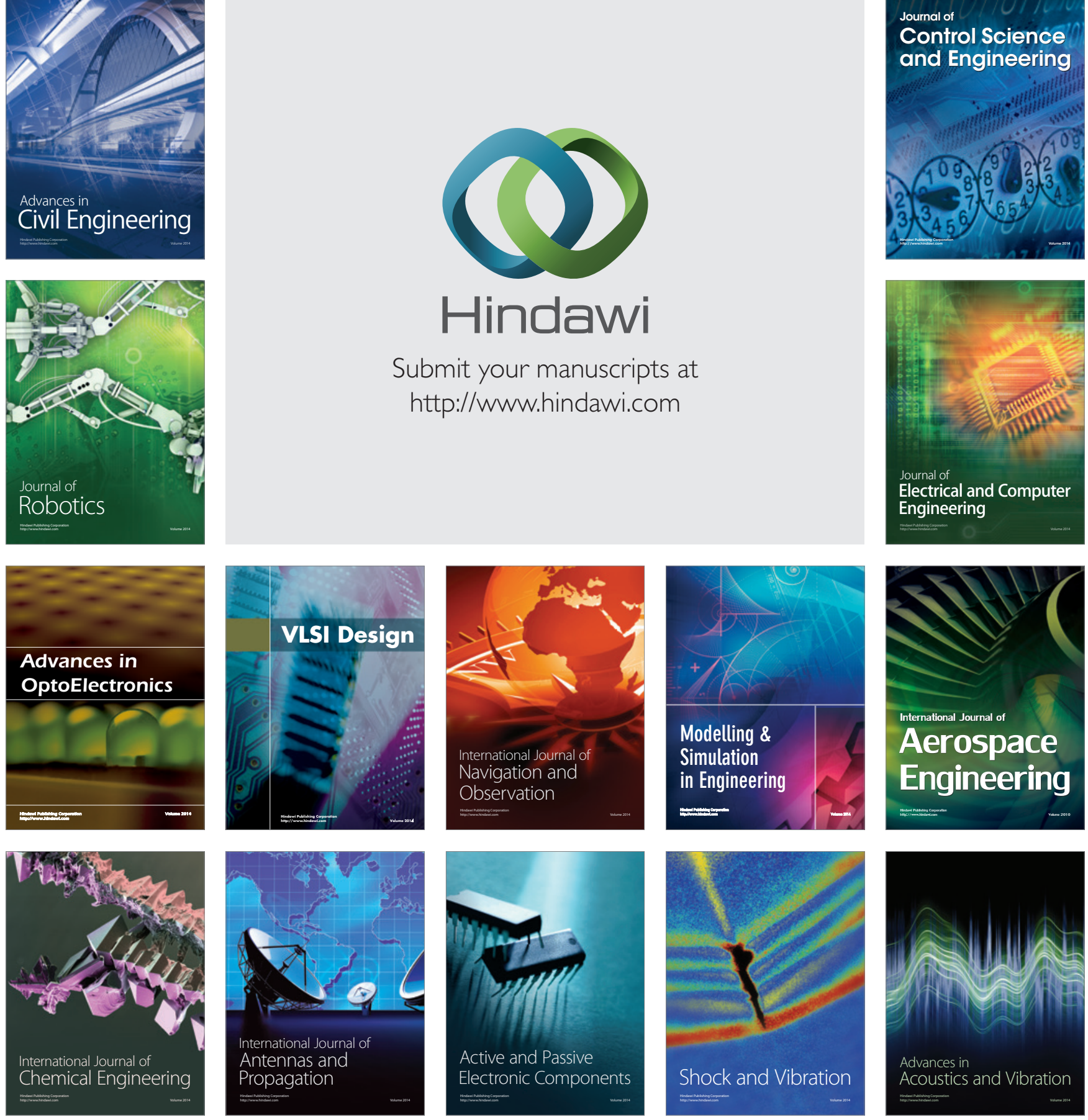